\title{
THE EFFECT OF TRAUMATIC EVENTS ON SOCIAL BEHAVIOR OF UNIVERSITY STUDENTS \\ Shahida Perveen ${ }^{1}$, Najma Iqbal Malik ${ }^{2 *}$ \\ ${ }^{1}$ Lecturer, Department of Psychology, University of Sargodha, Pakistan ${ }^{2 *}$ Associate Professor, Department of Psychology, University of Sargodha, Pakistan. \\ Email: ${ }^{1}$ shahidaperveen11@gmail.com, ${ }^{2 *}$ najmamalik@gmail.com
}

Article History: Received on $25^{\text {th }}$ April 2021, Revised on $11^{\text {th }}$ June 2021, Published on $15^{\text {th }}$ June 2021

\begin{abstract}
Purpose of the study: The present study aims at measuring the effect of traumatic events on social behavior among university students.

Methodology: Purposive sample of $n=200$ students was selected from various universities of Punjab, Pakistan. Impact of events scale-revised (Weiss, 2007), and Texas social behavior inventory (Helmreich \& Stapp, 1974) were used to measure study variables. Pearson correlation was carried out to explore correlations. Further, the prediction was checked through regression analysis. Additional analysis includes a t-test to check gender differences.
\end{abstract}

Main Findings: Significant differences were found in adolescents as compared to young adults on the scale of the traumatic events. Correlation analysis indicated a significant negative relationship between traumatic events (intrusion, avoidance, and hyper-arousal) and social behavior (i.e., self-esteem). Further multiple regression analysis showed that traumatic events were a significant negative predictor of social behavior as traumatic events harm an individual's social behavior. Additionally, the t-test indicated non-significant gender differences.

Applications of this study: These research results have implications in academic settings, clinical and counseling. And it helps to improve overall social behavior at the individual level, which ultimately serves to ensure a better community and sound environment.

Originality/Uniqueness of present investigation: An empirical investigation of the phenomenon observed among human participants' natural behavior with no manipulation.

Keywords: Traumatic Events, Social Behavior, Psychological Health, Trauma Impact, University Students.

\section{INTRODUCTION}

The experience of trauma among students is inflated all over the globe during the previous decade. Teachers and students were equally affected by traumatic experiences (Bohannon et al., 2019). Complex trauma is a consequence of persistent or recurrent exposure to the same trauma for a long period, which more commonly results in serious dysfunction or lowered well-being. (Thomas et al., 2019).

The underlying reasons for psychological and emotional trauma occur in daily life, including accidents in traffic, diseases, falls, rape, violence in school, and domestic settings - additionally, catastrophic events like terrorist attacks and wars, and natural disasters. Exposure to trauma from an individual perspective can become very serious, and it started to intervene with his capability to continue his normal life. On the other hand, community-level impacts of trauma will also prove to be disastrous. It usually changes the basic social as well as physical fabric within that community (like the tsunamis in Asia, earthquakes within Haiti and China, moreover, on the Gulf Coast of the U.S. the Hurricane Katrina occurred, floods in Pakistan and Bangladesh, the terrorist attacks observed in Kampala, Bagdad, London, Kabul, Madrid, and New York City) (Galea et al., 2002 \& 2007).

Ginwright (2021) mentioned the importance of how we viewed young people. Youth development offered an important shift in focus from viewing youth as problems to be solved to community assets that simply required support and healthy development opportunities. Further, Ginwright (2021) mentioned that practitioners and policy stakeholders had recognized the impact of trauma on learning and healthy development. On the other hand, Souers (2018) recommends six specific strategies that help teachers reach students whose lives are affected by trauma. Malik et al. (2018), the assumption that exposure to continuous terrorism is sensitizing was partially supported in Pakistani culture.

Further, Shah et al. (2020) highlight the severity of the impact of trauma on regional journalists in Pakistan. Exposure to work-related trauma, active emotional coping, and avoidant emotional coping were statistically significant factors associated with PTSD symptoms. Another study by Amin et al. (2020) demonstrates that SSET, delivered by a clinically trained provider, may be an effective intervention for treating traumatic stress symptoms among children affected by natural disasters like flooding, particularly in under-resourced contexts. Further, Waldman (2019) mentioned the importance of this natural human response to threats: it helps individuals react to potentially dangerous situations and not spend time determining whether a threat is real or not. "It's wonderful if you're in the forest and you see a bear, and you need to act," says Lamb. "It's terrible in a classroom or a hallway."

In the DSM-IV-TR (American Psychiatric Association [APA], 2000), within the diagnostic criteria of Post-traumatic stress disorder (PTSD), the traumatic event in Criterion A was defined as an individual "experienced, witnessed, or was 
confronted with an event or events that involved actual or threatened death or serious injury, or a threat to the physical integrity of self or others" (APA, 2000). Prevalence rates of trauma are greatly affected by how these trauma's definitions and their assessment. Frazier et al. (2009) highlighted around 10 studies whose focus was the assessment of trauma prevalence in the lifetime of college and university students of the United States. These all studies showed that most of the students' population experienced a traumatic event at some point in their life (Frazier et al., 2009). Regardless of their semester, university and college-level students faced many stressors, which can be defined as trauma for some people and not for others. During the previous two years, students have admitted trauma experiences even within office hours and during classes. Their stressful events at a personal level ranged from financial problems to unplanned pregnancy or the loss of a parent. It further includes homelessness, serious injury, or long-term disease. Few of these major events could impact their lives in the long run. Students with such issues may seem withdrawn, increased absenteeism, late or absent from submitting their assignments, and do not ask for any kind of support from others (Bohannon et al., 2019).

World mental health Surveys in 24 countries $(n=68,894)$ conducted an assessment of 29 types of traumas in a lifetime and evaluated PTSD two times for each participant. Firstly, for the 'worst' trauma in their lifetime and secondly, separately assessed any trauma randomly out of these 29 traumas to explore the individual differences in traumatic experiences. They mentioned in their results that a total of $70.4 \%$ of participants had exposure to some trauma in their lifetime, with an average of 3.2 traumas per individual. Considerable differences among kinds of trauma were obtained at the start of PTSD, but perseverance was low in these individuals. Traumas regarding interpersonal violence reported the highest risk. The proportions of each trauma type were mentioned as rape (13.1\%), unexpected death of a loved one $(11.6 \%)$, being stalked $(9.8 \%)$, and other sexual assault $(15.1 \%)$. The death of a loved one is reported as very common and at low risk of PTSD. The other three were mentioned as uncommon traumas comparably and at high risk of PTSD.

Further, intimate partner sexual violence was reported as a wide category, and it put $42.7 \%$ of people at risk of PTSD. Moreover, the previous history of trauma leads to exposure to trauma in the future as well as increased risk of PTSD in the future. (Kessler et al., 2017). Another study reported the prevalence of exposure to trauma and the psychological effects of traumatic events in 983 university graduates in Israel. The psychological impact of the experience of single vs. multiple events, which are considered traumatic, along with the impacts of physical injuries caused by these traumas, was explored. In this study, $67 \%$ of the participants reported their exposure to a traumatic event at least once in their life, while only 6\% of them further diagnosed with PTSD in the future (Amir \& Sol, 1999).

\section{Social behavior}

Bohannon et al. (2019) mentioned that our communities and schools reported growth in violence as well as unrest which lead to growing interest in educational leadership. College students were exposed to traumatic events with a cognitivebehavioral demonstration of the phenomenon of learned helplessness, work avoidance as well as less self-efficacy. Additionally, with the help of teachers, students can also be reached other than college classroom settings to support those struggling regarding college courses due to their traumatic experiences. Further, Research mentioned that recurrent exposure to the same trauma for a long period in childhood is linked with impairment in students' performance in school. For example, their social, cognitive development, emotional, as well as brain development can be significantly impaired by exposure to traumatic stress (Perfect et al., 2016). Trauma in childhood hurts students' self-regulation, comprehension, organization, and memorization, which further affect their academic performance and social experiences throughout their school life. (Thomas et al., 2019).

Muldoon et al. (2019) presented a review to emphasize the role of social factors among outcomes followed by trauma. They discussed it in two ways. Firstly, to enhance the importance of social identity framework in understanding the effect and experience of psychological trauma. Secondly, to conclude based on the Social Identity Model of Identity Change (SIMIC) for understanding the individual's responses towards trauma. They concluded that trauma outcomes could cause a change in an individual's social identity as well as its resources.

In light of these empirical bases, present exploration was designed because trauma can deeply impact educational settings (Espelage et al., 2011). Moreover, interaction with such an event that is perceived as trauma by an individual can alter his capability to arrange educational tasks priority-wise and practically attempt such educational tasks in the long term after the trauma experience (Bohannon et al., 2019). A significant amount of literature was available in Pakistani culture focusing on different traumatic events separately (Malik et al., 2018; Bibi et al., 2019; Kanwal \& Jami, 2019; Ahmed et al., 2020). But no previous studies focused on the overall impact of traumatic events on social behavior. So present study was designed to fulfill this gap in indigenous settings. Keeping in view this literature, the present study had the specific objectives to see the relationship and impact of traumatic events on students' social behavior and explore the gender differences among traumatic events and social behavior of students. The following hypotheses were formulated to achieve the objectives mentioned above:

H1: There will be a positive relationship between traumatic events (and their subscales, i.e., intrusion, avoidance, and hyperarousal) and social behavior.

H2: Traumatic events and their subscales will be significant predictors of social behavior. 
H3: There will be significant gender differences in traumatic events and social behavior.

H4: There will be significant age differences in traumatic events and social behavior.

\section{METHODOLOGY}

Sample

A cross-sectional survey research design with a purposive sample of 200 university students comprised of both male $(n=$ 96) and female $(n=104)$ was selected from five different Public sector universities of Punjab, Pakistan was used in this study. As inclusion criteria, specifically, the university enrolled regular students with the age range of 15 years to 30 years $(\mathrm{M}=22.56, \mathrm{SD}=2.69)$ were included in the study. College students of the same age or students who are not enrolled in any university were excluded. The education of the sample was specified as graduate (30\%), master's degree holder (36\%), postgraduate (34\%), and they were living in urban areas (33\% students were living in a hostel and $77 \%$ day scholars). The study used a survey research design to collect data from the students of different universities in the Punjab province of Pakistan.

\section{Instruments}

A questionnaire booklet comprised of two standardized instruments and one demographic information sheet, and an informed consent form was used to collect data in the current study. The demographic information sheet included the pertinent information about the sample, age, gender, education level, residence, and institute sector (public/private).

The Impact of Event Scale-Revised Scale (IES-R) (Weiss, 2007). It is a concise and self-reported 22-item questionnaire. It further contains three subscales. i.e., intrusion (item number 1,2,3,6,9,14,16,20), avoidance (item number $5,7,8,11,12,13,17,22$ ), and hyper-arousal (item number 4,10,15,18,19,21). Respondents answered according to their present symptomatic stress level in the form of a Likert scale ranging from 0 to 4 concerning any traumatic event/s they experienced. This scale doesn't have any specific cut-off scores. The higher the scores, the greater the symptomatic stress; the scale had Chronbach's alpha $=0.92$, representing it as a reliable measure. Further, convergent as well as content validity was previously reported as satisfactory in Pakistani culture. Before administration, the respondents were instructed to recall any event they perceived as traumatic and experienced in the previous week that they feel terrifying or hurtful (Kerai et al., 2017).

Texas Social Behavior Inventory (TSBI) (Helmreich \& Stapp, 1974): It has sixteen questions that calculate individuals' self-esteem within social situations or their social competence. The items were about the student's level of selfconfidence among other people, the capability of dealing with strangers, and how much they feel comfortable in social gatherings. This scale with responses ranging from $1=$ strongly disagree up to $5=$ strongly agree. Higher scores indicated more self-esteem (Thompson, 2014).

\section{Procedure and data analysis}

The institutional research board approved the research, and all the ethical protocols of the APA research adhered to. A questionnaire booklet inclusive of informed consent, demographic data sheet form, and all the standardized instruments was designed and used for data collection from the study participants. The sample was approached after the formal approval of relevant authorities and was clarified regarding the purpose along with the objectives of this exploration. After obtaining the informed consent, proper instructions were given to them at the start of each form about how to fill this questionnaire. They were asked to record their honest responses, and the average time recorded was 15 minutes per participant. In the end, they were thanked for their voluntary participation. The total response rate was recorded to be $85 \%$, and collected data were analyzed through SPSS version 25 to test hypotheses and produce the results.

\section{RESULTS}

Results of the study were ascertained by suitable statistical analysis, i.e., the Alpha coefficient was computed to determine the soundness of instruments; Pearson correlation was done to assess the relationship between study variables (see table 1). The regression analysis was carried out to see the impact of trauma on the social behavior of students (see table 2), and a t-test was carried out to determine gender differences in terms of trauma and social behavior among students (see table 3).

Table 1: Pearson Correlation Matrix, Alpha Reliabilities and descriptives of All Study Variables for University students $(\mathrm{N}=200)$

\begin{tabular}{lllllllll}
\hline Variables & $\boldsymbol{M}$ & $\boldsymbol{S D}$ & $\mathbf{1}$ & $\mathbf{2}$ & $\mathbf{3}$ & $\mathbf{4}$ & $\mathbf{5}$ & $\boldsymbol{\alpha}$ \\
\hline 1. Traumatic event & 40.42 & 15.23 & - & $.19^{* *}$ & $.93^{* *}$ & $.91^{* *}$ & $.88^{* *}$ & .92 \\
\hline 2. Social behavior & 46.49 & 8.76 & - & - & $.19^{* *}$ & $.17^{*}$ & $.16^{*}$ & .81 \\
\hline 3. Intrusion & 14.56 & 6.07 & - & - & - & $.77^{* *}$ & $.76^{* *}$ & .82 \\
\hline 4. avoidance & 15.07 & 5.81 & - & - & - & - & $.68^{* *}$ & .68 \\
\hline 5. Hyperarousal & 10.79 & 4.85 & - & - & - & - & - & .79 \\
\hline
\end{tabular}

$* * p<.01$. 
Source: Authors

Table 1 represented Cronbach's alpha values of scales and subscales along with Pearson correlation among study variables. It showed that the traumatic events scale and its subscales (i.e., intrusion, avoidance, and hyperarousal) were significantly related to social behavior, which means more traumatic events, more effects on social behavior. Additionally, all scales were reliable measures of the constructs.

Table 2: Linear and Multiple Regression Predicting Social Behavior among University students (N=200)

\begin{tabular}{llll}
\hline Model & \multicolumn{3}{l}{ Social behavior } \\
\hline & $\boldsymbol{B}$ & $\Delta \boldsymbol{R}^{2}$ & $\mathbf{F}$ \\
\hline Traumatic event sum & $.19^{* *}$ & .04 & $7.81^{* *}$ \\
\hline Intrusion & $.11^{* *}$ & & \\
\cline { 1 - 2 } Avoidance & $.06^{* *}$ & .04 & $2.61^{*}$ \\
\cline { 1 - 2 } Hyperarousal & $.04^{* *}$ & & \\
\hline
\end{tabular}

$* * \mathrm{p}<.01$.

\section{Source: Authors}

In Table 2, linear regression was done separately with the total score of the traumatic event, while for its subscales, multiple regression was carried out in SPSS. It represented that overall traumatic events were a significant predictor of social behavior at $[\mathrm{F}(1,199)=7.81, \mathrm{p}<.01]$ and explains $4 \%$ variance in social behavior. Additionally, this table represented the subscales of traumatic events separately (i.e., intrusion, avoidance, and hyperarousal) were also significant predictors of social behavior.

Table 3: Mean, Standard Deviation and t-values for male and female students on traumatic events and social behavior

$$
(\mathrm{N}=200)
$$

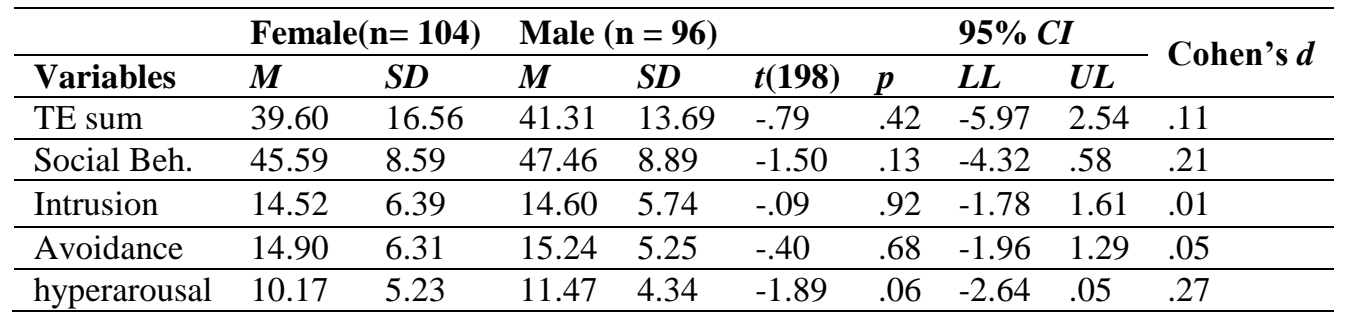

Note. $\mathrm{TE}=$ Traumatic events sum; Social Beh. $=$ social behavior

\section{Source: Authors}

Table 3 presented gender differences through a t-test among traumatic events and their subscales (i.e., intrusion, avoidance, and hyper-arousal) and social behavior. Gender differences on all other scales and subscales were found nonsignificant.

Table 4: Mean, Standard Deviation and $\mathrm{t}$-values for the age of students on traumatic events and social behavior $(\mathrm{N}=$ 200)

\begin{tabular}{|c|c|c|c|c|c|c|c|c|c|}
\hline \multirow[b]{2}{*}{ Variables } & \multicolumn{2}{|c|}{$\begin{array}{l}\text { Adolescents } \\
(15-20 \text { years }) \\
(n=104)\end{array}$} & \multicolumn{2}{|c|}{$\begin{array}{l}\text { Young adults } \\
(20-25 \text { years }) \\
(n=96)\end{array}$} & \multirow[b]{2}{*}{$t(\mathbf{1 9 8})$} & \multicolumn{3}{|c|}{$95 \% C I$} & \multirow[t]{2}{*}{ Cohen's $d$} \\
\hline & $M$ & $S D$ & $M$ & $S D$ & & $p$ & $L L$ & $U L$ & \\
\hline TE sum & 37.15 & 15.29 & 43.71 & 14.29 & $-3.11 * *$ & .002 & -10.7 & -2.40 & .44 \\
\hline Social Beh. & 46.50 & 8.04 & 46.44 & 9.59 & 0.47 & .96 & -2.42 & 2.54 & .006 \\
\hline Intrusion & 13.15 & 5.99 & 16.00 & 5.79 & $-3.39 * *$ & .001 & -4.49 & -1.19 & .48 \\
\hline Avoidance & 14.16 & 5.94 & 15.92 & 5.43 & $-2.16^{*}$ & .03 & -3.35 & -0.15 & .31 \\
\hline hyperarousal & 9.84 & 5.14 & 11.79 & 4.33 & $-2.91 * *$ & .004 & -3.27 & -0.63 & .41 \\
\hline
\end{tabular}

Note. $\mathrm{TE}=$ Traumatic events sum; Social Beh. $=$ social behavior

$* * \mathrm{p}<.01, * \mathrm{p}<.05$.

Source: Authors

Table 4 presented differences on the basis of the age of students through a t-test among traumatic events and their subscales (i.e., intrusion, avoidance, and hyper-arousal) and social behavior. The table showed significant differences in adolescents and young adults on the scale of the traumatic event and its all subscales, i.e., intrusion, avoidance, and 
hyper-arousal. Young adults score high on traumatic events and all subscales in comparison with adolescents. Whereas age differences in social behavior were found non-significant.

\section{DISCUSSION/ANALYSIS}

Trauma can deeply impact educational settings (Espelage et al., 2011); moreover, interaction with such event which is perceived as trauma by an individual, can alter his capability of arranging educational tasks priority wise and practically attempt such educational tasks in the long term after the experience of trauma (Bohannon et al., 2019).

In academic settings, trauma-informed education, which can also be termed as trauma-informed practices, demands administration support, especially trauma-sensitive designed activities in the classroom, restorative and positive feedback, procedures and policies mended, teacher as well as staff training in professional development, and strong collaboration among mental health professionals and school staff (Oehlberg, 2008). These recommendations are supposed to improve students' performance along with retention and improvement in a climate of educational institutions. (Thomas et al., 2019).

The present study was conducted to explore the relationship of traumatic events with social behavior among university students. Pearson correlation was carried out to explore correlations. Further, the prediction was checked through regression analysis. Additional analysis includes a t-test to check gender differences. Reliability analysis was done to ensure that these scales are reliable measures of constructs used in the present study.

The first hypothesis was stated as "there will be a positive relationship between traumatic events (and it's all subscales, i.e., intrusion, avoidance, and hyperarousal) and social behavior." This hypothesis was proved significant and hence accepted in the present study. This was in line with previous literature as Muldoon et al. (2019) presented a review to emphasize the role of social factors among outcomes followed by trauma. They discussed it in two ways. Firstly, to enhance the importance of social identity framework in understanding effect and experience of psychological kind of trauma. Secondly, to conclude on the basis of the Social Identity Model of Identity Change (SIMIC) for understanding the individual's responses towards trauma. They concluded that trauma outcomes could cause a change in an individual's social identity as well as its resources. Hence, we can conclude our study to support the argument of the present study that traumatic events had significant positive correlations with social behavior.

In another study by Muldoon et al. (2019), it was mentioned that individuals became more resilient in the contempt of trauma if their social identities, which are very important to them, are maintained by showing resilience, or when they can develop new social identities. Further, they mentioned that where old (or new) positive social identities are rejuvenated or expand to the self, this provides a basis for growth after facing the trauma. Another study by Bohannon et al. (2019) mentioned that our communities and schools reported growth in violence as well as unrest which lead to growing interest in educational leadership. College students were exposed to traumatic events with a cognitivebehavioral demonstration of the phenomenon of learned helplessness, work avoidance as well as less self-efficacy. Additionally, with the help of teachers, students can also be reached other than college classroom settings to support those struggling regarding college courses due to their traumatic experiences.

The second hypothesis stated that traumatic events would predict social behavior. This was also accepted. Results in table 2 indicated traumatic events and all its subscales were significant predictors of social behavior and explained a $4 \%$ variance in social behavior. These findings were in line with previous literature as the study by Amir and Sol (1999) reported the prevalence of exposure to trauma and the psychological effects of traumatic events in 983 university graduates in Israel. The psychological impact of the experience of single vs. multiple events considered traumatic, and the impacts of physical injuries caused by these traumas were explored. In this study, $67 \%$ of the participants reported their exposure to a traumatic event at least once in their lives, while only $6 \%$ of them were further diagnosed with PTSD in the future.

The third hypothesis compares the gender differences in terms of traumatic events and social behavior. The present study results revealed non-significant gender differences. This finding contrast with previous studies as Frazier et al. (2009) reported men and women had significant differences in exposure to 8 out of 23 traumatic events. One reason for these significant differences reported by the author was more women participants (73\%) compared to men participants (27\%) and because of the difference in exposure to events in men and women. Another study mentioned that men reported a higher risk of exposure to traumatic events, whereas women reported a higher risk of PTSD (Amir \& Sol, 1999).

The last hypothesis was a comparison of adolescents with young adults on traumatic events and social behavior. Results indicated significant differences in traumatic events whereas non-significant differences in social behavior. This is evident from the fact that an increase in age causes an increase in exposure to new trauma in addition to the negative impact of their previous experiences of trauma to add up in severity of problem and vulnerability of facing further problems. These results were in line with previous research as previously highlighted within the representative sample of various ages. For children of ages 2 to 17 years, 13.9 percent had experienced at least one disaster within their lifetime, whereas 4.1 percent from the sample mentioned the experience of at least one disaster within the previous year (BeckerBlease et al., 2010). The age of a person at exposure to trauma may also function as a risk factor in any disaster outcome. 
(Behavioral Health Report, 2018). Older age was also found to be a risk factor for tobacco, alcohol, and substance use following tornado exposure, as were prior traumatic events (Danielson et al., 2017).

These findings highlighted the need for trauma-informed care practices among students. According to Ginwright (2018), trauma-informed care broadly refers to a set of principles that guide and direct how we view the impact of severe harm on young people's mental, physical, and emotional health. Trauma-informed care encourages support and treatment to the whole person, rather than focusing on only treating individual symptoms or specific behaviors. Further, Hoover et al. (2018) describe the implementation and outcomes of Cognitive Behavioral Intervention for Trauma in Schools (CBITS); they mentioned that children demonstrated significant reductions in child posttraumatic stress disorder (PTSD) symptoms and problem severity and increases in child functioning.

\section{CONCLUSION}

The study concluded that traumatic events were related and were found significant predictors of social behavior among university students. Trauma can profoundly impact educational environments, and exposure to perceived trauma can impact an individual's ability to prioritize and attend to educational tasks long after the trauma has occurred. Few of the findings of the present study were contrary to previous literature as the present study reported no gender differences were found in traumatic events and social behavior. This finding contrast with previous studies, which reported men and women had significant differences in exposure to 8 out of 23 traumatic events.

\section{LIMITATION AND STUDY FORWARD}

The present study has few reservations. Firstly, the small sample size and various other influencing factors such as personality, coping strategies, education level, and socioeconomic status were beyond the scope of this study to check their effect on the phenomenon under investigation. This may impact the generalizability of the research results. So it is suggested that future research should incorporate the aspects mentioned above as main variables or demographics to check more precise and in-depth understating of these constructs.

Traumatic events are very uncertain and can occur at any time to anyone. No one can ensure his/her safety and can become a victim of some kind of traumatic experience. So understanding this phenomenon and research evidence can lead a person to conceptualize the issue in no time. This further leads him to save himself from the further disaster of that trauma and make himself psychologically strong and prepared to deal with it. So, these research results have implications in academic settings, in clinical and counseling of students. And it helps improve overall social behavior at the individual level, which ultimately serves to ensure a better community and sound environment. Future research should focus on the implementation of Cognitive Behavioral Intervention for Trauma in Schools (CBITS) mentioned by Hoover et al. (2018) and Bounce Back intervention mentioned by Santiago et al. (2018) in a university student sample.

\section{ACKNOWLEDGEMENT}

The voluntary joining in the project and student's cooperation is highly acknowledged. The authors further announce no financial support was related to the submission.

\section{DECLARATION OF COMPETING INTEREST}

The authors declare no conflicts of interest.

\section{AUTHORS CONTRIBUTION}

The study was conceptualized majorly by Shahida Perveen. Moreover, the collection of data, data analysis along the study's literature review was done by the same. Further, Co-author Najma Iqbal Malik supervised the research process, contributed to data analysis, reviewed and finalized the paper. All authors have co-written and approved the final version of the manuscript.

\section{REFERENCES}

1. Ahmed, W., Ahmad, A., \& Bhatti, M. B. (2020). Bonding, Academic Culture, and Aggressive Behavior among University Students in Pakistan. Global Regional Review, V(I), 8-14. https://doi.org/10.31703/grr.2020(V-I).02

2. American Psychiatric Association (2000). Diagnostic and statistical manual of mental disorders (4th ed., text rev.). Washington, DC: Author.

3. Amin, R., Nadeem, E., Iqbal, K., Asadullah, M. A., \& Hussain, B. (2020). Support for Students Exposed to Trauma (SSET) Program: An Approach for Building Resilience and Social Support Among Flood-Impacted Children. School Mental Health, 12(3), 493-506. https://doi.org/10.1007/s12310-020-09373-y

4. Amir, M., \& Sol, O. (1999). Psychological impact and prevalence of traumatic events in a student sample in Israel: The effect of multiple traumatic events and physical injury. Journal of Traumatic Stress: Official Publication of The International Society for Traumatic Stress Studies, 12(1), 139-154. https://doi.org/10.1023 /A:1024754618063

5. Becker-Blease, K. A., Turner, H. A., \& Finkelhor, D. (2010). Disasters, victimization, and children's mental health. Child Development, 81(4), 1040-1052. https://doi.org/10.1111/j.1467-8624.2010.01453.X 
6. Behavioural Health Conditions in Children and Youth Exposed to Natural Disasters (2018). SAMHSA Disaster Technical Assistance Center Supplemental Research Bulletin. 1-20.

7. Bernat, J. A., Ronfeldt, H. M., Calhoun, K. S., \& Arias, I. (1998). Prevalence of traumatic events and peritraumatic predictors of posttraumatic stress symptoms in a non-clinical sample of college students. Journal of Traumatic Stress, 11, 645-664. https://doi.org/10.1023/A:1024485130934

8. Bibi, A., Blackwell, S. E., \& Margraf, J. (2019). Mental health, suicidal ideation, and experience of bullying among university students in Pakistan. Journal of Health Psychology. https://doi.org/10.1177/1 $\underline{359105319869819}$

9. Bibi, A., Lin, M., \& Margraf, J. (2020). Salutogenic constructs across Pakistan and Germany: A cross-sectional study. International Journal of Clinical and Health Psychology, 20(1), 1-9. https://doi.org/10.1016/j.ij chp.2019.10.001

10. Bohannon, L., Clapsaddle, S., \& McCollum, D. (2019). Responding to College students who exhibit adverse manifestations of stress and trauma in the college classroom. In FIRE: Forum for International Research in Education, 5(2). https://doi.org/10.32865/fire201952164

11. Danielson, C. K., Cohen, J. R., Adams, Z. W., Youngstrom, E. A., Soltis, K., Amstadter, A. B., \& Ruggiero, K. J. (2017). Clinical decision-making following disasters: Efficient identification of PTSD risk in adolescents. Journal of Abnormal Child Psychology, 45(1), 117-129. https://doi.org/10.1007/s10802-016-0159-3

12. Espelage, D., Anderman, E. M., Brown, V. E., Jones, A., Lane, K. L., McMahon, S. D. \& Reynolds, C. R. (2011). Understanding and preventing violence directed against teachers. American Psychological Association, 68(2), 75-87. https://doi.org/10.1037/a0031307

13. Frazier, P., Anders, S., Perera, S., Tomich, P., Tennen, H., Park, C., \& Tashiro, T. (2009). Traumatic events among undergraduate students: Prevalence and associated symptoms. Journal of Counseling Psychology, 56(3), 450. https://doi.org/10.1037/a0016412

14. Galea, S., Brewin, C.R., Gruber, M., et al. (2007). Exposure to hurricane-related stressors and mental illness after Hurricane Katrina. Archives of General Psychiatry, 64, 1427-1434. https://doi.org/10.1001/archpsyc.64 .12 .1427

15. Galea, S., Resnick, H., Ahern, J., Gold, J., Bucuvalas, M., Kilpatrick, D., Stuber, J, Vlahov, D. (2002). Posttraumatic stress disorder in Manhattan, New York City, after the September 11th terrorist attacks. Journal of Urban Health: Bulletin of the New York Academy of Medicine, 79(3), 340-53. https://doi.org/1 $0.1093 /$ jurban/79.3.340

16. Ginwright, S. (2018). The future of healing: Shifting from trauma-informed care to healing centred engagement. Retrieved from https://medium.com/@ginwright/the-future-of-healing-shifting-from-trauma-informed-care-tohealing-centered-engagement-634f557ce69c

17. Ginwright, S. (2021). The Future of Healing: Shifting From Trauma-Informed Care to Healing-Centered Engagement. Retrieved from: https://youthrex.com/blog/the-future-of-healing-shifting-from-trauma-informedcare-to-healing-centered-engagement/

18. Green, B. L., Goodman, L. A., Krupnick, J. L., Corcoran, C. B., Petty, R. M., Stockton, P., \& Stern, N. M. (2000). Outcomes of single versus multiple trauma exposure in a screening sample. Journal of Traumatic Stress, 13, 271-286. https://doi.org/10.1023/A:1007758711939

19. Helmreich, R., \& Stapp, J. (1974). Short forms of the Texas Social Behavior Inventory (TSBI), an objective measure of self-esteem. Bull. Psychon. Soc. 4, 473-475. https://doi.org/10.3758/BF03334260

20. Hoover, S. A., Sapere, H., Lang, J. M., Nadeem, E., Dean, K. L., \& Vona, P. (2018). Statewide implementation of an evidence-based trauma intervention in schools. School Psychology Quarterly, 33(1), 44-53. https://doi.org/10.1037/spq0000248

21. Kanwal, H., \& Jami, H. (2019). Exploring Modes, Strategies, and Psychosocial Consequences of Cyberbullying Perpetration and Victimization Among University Students. Pakistan Journal of Psychological Research, 787817. https://doi.org/10.33824/PJPR.2019.34.4.43

22. Kerai, S. M., Khan, U. R., Islam, M., Asad, N., Razzak, J., \& Pasha, O. (2017). Post-traumatic stress disorder and its predictors in emergency medical service personnel: a cross-sectional study from Karachi, Pakistan. BMC Emergency Medicine, 17(1), 1-7. https://doi.org/10.1186/s12873-017-0140-7

23. Kessler, R. C., Aguilar-Gaxiola, S., Alonso, J., Benjet, C., Bromet, E. J., Cardoso, G. \& Koenen, K. C. (2017). Trauma and PTSD in the WHO world mental health surveys. European Journal of Psychotraumatology, 8(sup5), 1353383. https://doi.org/10.1080/20008198.2017.1353383

24. Malik, O. F., Schat, A. C. H., Raziq, M. M., Shahzad, A., \& Khan, M. (2018). Relationships between perceived risk of terrorism, fear, and avoidance behaviors among Pakistani university students: A multigroup study. Personality and Individual Differences, 124, 39-44. https://doi.org/10.1016/j.paid.2017.11.044

25. Muldoon, O. T., Haslam, S. A., Haslam, C., Cruwys, T., Kearns, M., \& Jetten, J. (2019). The social psychology of responses to trauma: Social identity pathways associated with divergent traumatic responses. European Review of Social Psychology, 30(1), 311-348. https://doi.org/10.1080/10463283.2020.1711628

26. Oehlberg, B. (2008). Why Schools Need to Be Trauma Informed. Trauma and Loss: Research and Interventions, 8(2), 1-4. 
27. Owens, G. P., \& Chard, K. M. (2006). PTSD severity and cognitive reactions to trauma among a college sample: An exploratory study. Journal of Aggression, Maltreatment, and Trauma, 13, 23-36. https://doi.org/10.1300/J146v13n02 02

28. Perfect, M. M., Turley, M. R., Carlson, J. S., Yohanna, J., \& Saint Gilles, M. P. (2016). School-related outcomes of traumatic event exposure and traumatic stress symptoms in students: A systematic review of research from 1990 to 2015. School Mental Health: A Multidisciplinary Research and Practice Journal, 8(1), 7-43. https://doi.org/10.1007/s12310-016-9175-2

29. Santiago, C. D., Raviv, T., Ros, A. M., Brewer, S. K., Distel, L. M., Torres, S. A., ... \& Langley, A. K. (2018). Implementing the Bounce Back trauma intervention in urban elementary schools: A real-world replication trial. School Psychology Quarterly, 33(1), 1. https://doi.org/10.1037/spq0000229

30. Scarpa, A., Fikretoglu, D., Bowser, F., Hurley, J. D., Pappert, C. A., Romero, N., \& Van Voorhees, E. (2002). Community violence exposure in university students. Journal of Interpersonal Violence, 17, $253-272$. https://doi.org/10.1177/0886260502017003002

31. Shah, S. F. A., Jan, F., Ginossar, T., McGrail, J. P., Baber, D., \& Ullah, R. (2020). Trauma exposure and posttraumatic stress disorder among regional journalists in Pakistan. Journalism, 1464884920965783. https://doi.org/10.1177/1464884920965783

32. Souers, K. (2018). Responding with care to students facing trauma. Educational Leadership, 75(4), 32-36.

33. Thomas, M. S., Crosby, S., \& Vanderhaar, J. (2019). Trauma-informed practices in schools across two decades: An interdisciplinary review of research. Review of Research in Education, 43(1), 422-452. https://doi.org/10.3 102/0091732X18821123

34. Thompson, T. (2014). Personal and Vicarious Embarrassability. Journal of Individual Differences, 35(1) 1-22. https://doi.org/10.1027/1614-0001/a000122

35. Vrana, S., \& Lauterbach, D. (1994). Prevalence of traumatic events and post-traumatic psychological symptoms in a non-clinical sample of college students. Journal of Traumatic Stress, 7, 289-302. https://doi.org/10.1002/jt $\underline{\text { s.2490070209 }}$

36. Waldman, C. (2019). Podcast: The Impact of Trauma on Student Learning. Retrieved from: https://all4ed.org/podcast-the-impact-of-trauma-on-student-learning/

37. Watson, S. B., \& Haynes, S. N. (2007). Brief screening for traumatic life events in female undergraduate health service patients. International Journal of Clinical and Health Psychology, 7, 261-282.

38. Weiss, D. S. (2007). The Impact of Event Scale: Revised. In J. P. Wilson \& C. S.-k. Tang (Eds.), International and cultural psychology. Cross-cultural assessment of psychological trauma and PTSD (p. 219-238). Springer Science + Business Media. https://doi.org/10.1007/978-0-387-70990-1_10 